\title{
A REMARK ON A THEOREM OF VO VAN TAN
}

\author{
MIHNEA COLTOIU
}

\begin{abstract}
In this paper we consider the following problem: Let $(X, S)$ be a 1-convex manifold with 1-dimensional exceptional set $S$. Does it follow that $X$ is a Kähler manifold?

Although this was answered in the affirmative by Vo Van Tan in two papers, we show that his proofs are wrong. It is also shown that the Kähler condition implies that any strongly pseudoconvex domain $D \Subset X$ is embeddable, i.e. can be realized as a closed analytic submanifold in some $\mathbf{C}^{N} \times \mathbf{P}_{M}$.

On the other hand it is known that under some additional assumptions on $S$ ( $S$ is not rational or $S \simeq \mathbf{P}^{1}$ and $\operatorname{dim} X \neq 3$ ) it follows that $X$ is embeddable, in particular it is Kählerian.
\end{abstract}

In [3] Vo Van Tan proved the following result:

THEOREM 1. Let $X$ be a 1-convex manifold with exceptional set $S$. Assume that $\operatorname{dim} S=1$. Then $X$ is Kählerian.

As pointed out in [1] the proof given in [3] by Vo Van Tan is wrong. In [4] Vo Van Tan obtained a new proof for the above theorem. Unfortunately, as we shall see, the new proof is again wrong.

We keep his notations. Let $L_{1}=[D]$ and choose $L_{2}=$ the trivial line bundle which clearly satisfies (**) in [4]. From $L=L_{1} \otimes L_{2}^{N}>0$ on $(\hat{X} \backslash \hat{S}) \cup V \supset \hat{X} \backslash D$ we obtain $L_{1}>0$ on $\hat{X} \backslash D$. Since $L_{1}$ is trivial and $>0$ on $\hat{X} \backslash D$ we obtain that there exists a strongly plurisubharmonic function $\psi: \hat{X} \backslash D \rightarrow \mathbf{R}$. Because $D$ can be contracted to a point it follows easily (from the extension theorem for plurisubharmonic functions) that there exists a plurisubharmonic function $\tilde{\psi}: \hat{X} \rightarrow$ $[-\infty, \infty)$ such that $\left.\tilde{\psi}\right|_{\hat{X} \backslash D}=\psi$. Since $T$ is compact it follows from the maximum principle that $\left.\tilde{\psi}\right|_{T}=$ constant, hence $\left.\psi\right|_{T \backslash T \cap D}=$ constant $(\neq-\infty)$. So $\left.\psi\right|_{T \backslash T \cap D}$ is not strongly plurisubharmonic and we get a contradiction.

It is not difficult to see where is the gap in Vo Van Tan's proof:

Let $X$ be a complex manifold, $L$ a line bundle on $X$ and $D_{1}, D_{2} \subset X$ open subsets. If $\left.L\right|_{D_{1}}$ is (semi)positive and $\left.L\right|_{D_{2}}$ is (semi)positive it does not follow that $\left.L\right|_{D_{1} \cup D_{2}}$ is (semi)positive.

In the case when $S$ satisfies some additional assumptions a stronger result was proved in [1]. Namely one has the following theorem.

Received by the editors May 1, 1987 .

1980 Mathematics Subject Classification (1985 Revision). Primary 32C10, 53C55; Secondary 32C45, 32E05, 32E10, 32F 10, 32F 15 . 
THEOREM 2. Let $X$ be a 1-convex manifold with 1-dimensional exceptional set $S$. Assume that $S$ is irreducible and

(i) $S$ is not rational

or

(ii) $S \simeq P^{1}$ and $\operatorname{dim} X \neq 3$.

Then $X$ is embeddable, in particular it is Kählerian.

In fact, the following result will show us that for 1-convex manifolds with 1dimensional exceptional set, the Kähler condition is very strong. Precisely one has:

THEOREM 3. Let $X$ be a Kählerian 1-convex manifold with 1-dimensional exceptional set $S$. Then any strongly pseudoconvex domain $D \subset \subset X$ is embeddable.

ProOF. We may assume that $S$ is obtained in $D$. We choose a strongly pseudoconvex domain $D^{\prime}$ with smooth boundary such that $D \subset \subset D^{\prime} \subset \subset X$. Since $\bar{D}^{\prime}$ is locally contractible it follows that the cohomology groups $H^{i}\left(\bar{D}^{\prime}, \mathbf{Z}\right)$ are finitely generated and from $H^{i}\left(\bar{D}^{\prime}, D^{\prime} ; \mathbf{Z}\right)=0$ for any $i$ we get that $H^{i}\left(D^{\prime}, \mathbf{Z}\right)$ are also finitely generated. In particular $H^{2}\left(D^{\prime}, \mathbf{Z}\right)$ is finitely generated. If $\xi_{1}, \ldots, \xi_{k}$ is a set of generators for the free part of $H^{2}\left(D^{\prime}, \mathbf{Z}\right)$ then $\bar{\xi}_{1}, \ldots, \bar{\xi}_{k}$, the images of $\xi_{1}, \ldots, \xi_{k}$ in $H^{2}\left(D^{\prime}, \mathbf{R}\right)$, is a set of generators for $H^{2}\left(D^{\prime}, \mathbf{R}\right)$.

On $D^{\prime}$ we consider the exact sequence of sheaves

$$
0 \rightarrow \mathbf{Z} \rightarrow O \stackrel{\exp (2 \pi i \cdot)}{\longrightarrow} 0^{*} \rightarrow 0
$$

From the long exact sequence for cohomology we obtain the exact sequence

$$
H^{1}\left(D^{\prime}, 0^{*}\right) \stackrel{c}{\rightarrow} H^{2}\left(D^{\prime}, \mathbf{Z}\right) \rightarrow H^{2}\left(D^{\prime}, 0\right)
$$

Since $S$ is 1-dimensional it follows from [1, Lemma 1] that $H^{2}\left(D^{\prime}, 0\right)=0$; hence the map $c$ is surjective. We choose line bundles $F_{1}, \ldots, F_{k} \in H^{1}\left(D^{\prime}, O^{*}\right)$ such that $c\left(F_{i}\right)=\xi_{i}$. Considering hermitian metrics on each line bundle $F_{i}$ we see that there exist $\eta_{1}, \ldots, \eta_{k}(1,1)$ real and closed forms on $D^{\prime}$ which represents $\bar{\xi}_{1}, \ldots, \bar{\xi}_{k}$ in $H^{2}\left(D^{\prime}, \mathbf{R}\right)$.

Let $\omega$ be a Kähler form on $X$. From the above remarks we obtain that there exist real constants $\lambda_{1}, \ldots, \lambda_{k}$ such that

$$
\omega \sim \lambda_{1} \eta_{1}+\cdots+\lambda_{k} \eta_{k} \text { on } D^{\prime}
$$

If $\varepsilon>0$ is small enough it follows that $\omega-\alpha_{1} \eta_{1}-\cdots-\alpha_{k} \eta_{k}$ is a Kähler form on $D$ for $\alpha_{i} \in \mathbf{R},\left|\alpha_{i}\right|<\varepsilon$. We choose integers $t_{1}, \ldots, t_{k}, m(m>0)$ such that $\left|\lambda_{i}-t_{i} / m\right|<\varepsilon$. If we set $\tilde{\omega}=\omega-\left(\lambda_{1}-t_{1} / m\right) \eta_{1}-\cdots-\left(\lambda_{k}-t_{k} / m\right) \eta_{k}$ then $\tilde{\omega}$ is a Kähler form on $D$ and $\tilde{\omega} \sim t_{1} / m \eta_{1}+\cdots+t_{k} / m \eta_{k}$. Hence $\tilde{\tilde{\omega}}:=m \tilde{\omega}$ is a Kähler form on $D$ whose cohomology class is in the image of the natural map $H^{2}(D, \mathbf{Z}) \rightarrow H^{2}(D, \mathbf{R})$. It follows that $D$ is a 1-convex Hodge manifold and from [2, Theorem 2.6] $D$ is embeddable. The proof of Theorem 3 is complete. 


\section{REFERENCES}

1. M. Colţoiu, On the embedding of 1-convex manifolds with 1-dimensional exceptional set, Comment. Math. Helv. 60 (1985), 458-465.

2. T. Peternell, On strongly pseudoconvex Kähler manifolds, Invent. Math. 70 (1982), 157-168.

3. Vo Van Tan, Vanishing theorems and Kählerity for strongly pseudoconvex manifolds, Trans. Amer. Math. Soc. 261 (1980), 297-302.

4. __ Correction to "Vanishing theorems and Kählerity for strongly pseudoconvex manifolds", Trans. Amer. Math. Soc. 291 (1985), 379-380.

Department OF MAThematics, INCREST, BD. PĂCII 220, 79622 BUChaREST, ROMANIA 